\title{
Two types of phenomenal instructions for size and distance judgments of objects presented on a two-dimensional plane'
}

\author{
C. J. A. haYes AND W. L. KING ${ }^{2}$ \\ DALHOUSIE UNIVERSITY
}

Demand characteristics were studied in a simple situation in which observers made size and distance estimates of stimuli presented on a two-dimensional linear perspective drawing. Half the Ss were asked questions stressing phenomenal report. The other half were asked the same questions preceded by instructions stressing the objective nature of the stimulus configuration but requesting, nonetheless, phenomenal report. Instructions resulted in a significantly greater perspective size illusion but did not affect the distance estimates. No meaningful correlations between size and distance estimates were found.

It is well substantiated that observers can make judgments of the size and distance of objects depicted on two-dimensional linear perspective line drawings (Smith, 1958; Smith, Smith, \& Hubbard, 1958; Wohlwill, 1962). Moreover, it has been shown, at least for three-dimensional objects, that instructions may have profound influence on size and distance judgments (Epstein, 1963; Carlson, 1962; McCready, 1965; Carlson \& Tassone, 1967). For example, Epstein (1963) found that phenomenal, objective, perspective, and projective attitudes produced size matches which were veridical, overestimated, more overestimated, and underestimated, respectively. Furthermore, he reported that size and distance estimations were not systematically related.

The present study stems from informal observations made in our laboratory suggesting that observer biases, to some extent, were responsible for countermanding the phenomenal questions asked of the $S$ while he was viewing the two-dimensional screen. It appeared that the size and distance estimations of the stimuli tended, for some observers, to be more objective than phenomenal despite the use of questions stressing phenomenal judgments.

This study was performed in an attempt to substantiate these impresslons that observer bias influenced size and distance judgments of objects on a two-dimensional linear perspective drawing. Two main groups of $\mathrm{Ss}$ were employed. In one, an Instruction group, the $\mathrm{Ss}$ were informed of the objective nature of the stimulus configuration but asked to give phenomenal estimations. The other, a No Instruction group, did not receive information about the objective nature of the stimulus configuration. If, as we have proposed, the Ss were attempting to "outguess" the $E$ and avoid the perspective illusion of size and depth by attempting to ignore or counter its effects, then systematic differences should be observed between the size and distance estimates of the two groups. The group receiving instructions should report a larger perspective illusion than the group not receiving instructions.

\section{Subjects}

METHOD

Eighty Ss from undergraduate and graduate psychology courses at Dalhousie University participated.

\section{Apparatus}

A viewing screen with an aperture $2 \mathrm{~mm}$ in diameter was employed with all Ss viewing the stimuli through this artificial pupil by resting their heads against a foam rubber head rest. A tracing paper screen was positioned between the $S$ and a $35 \mathrm{~mm}$ slide projector such that the projected image was directly in line with the artificial pupil. The screen was located $152.5 \mathrm{~cm}$ from the artificial pupil.

Two slides were employed, both of which contained two identical gray disks $4.5 \mathrm{~cm}$ in diameter, each thereby subtending $1.69^{\circ}$ of visual angle. The aperture limited the field of view to a visual angle of approximately $10.52^{\circ}$. These disks were arranged along a $75^{\circ}$ diagonal from the horizontal. The upper disk sat approximately along the center line of the drawing with the lower disk seen to the S's right. The disks were separated by $9.39^{\circ}$ of visual angle.

On one slide the two gray disks were superimposed on a linear perspective gradient composed of both vertical and horizontal lines as shown in Fig. 1. In the other, the disks were superimposed on a homogeneous light background.

\section{Procedure}

The Ss were randomly assigned to one of four groups, each containing 20 Ss. These were the Instruction-Perspective, the Instruction-No Perspective, the No Instruction-Perspective, and the No Instruction-No Perspective groups. Each $S$ was asked to estimate both the apparent size and distance of both gray disks. The order in which the Ss made their size and distance judgments was systematically varied. 


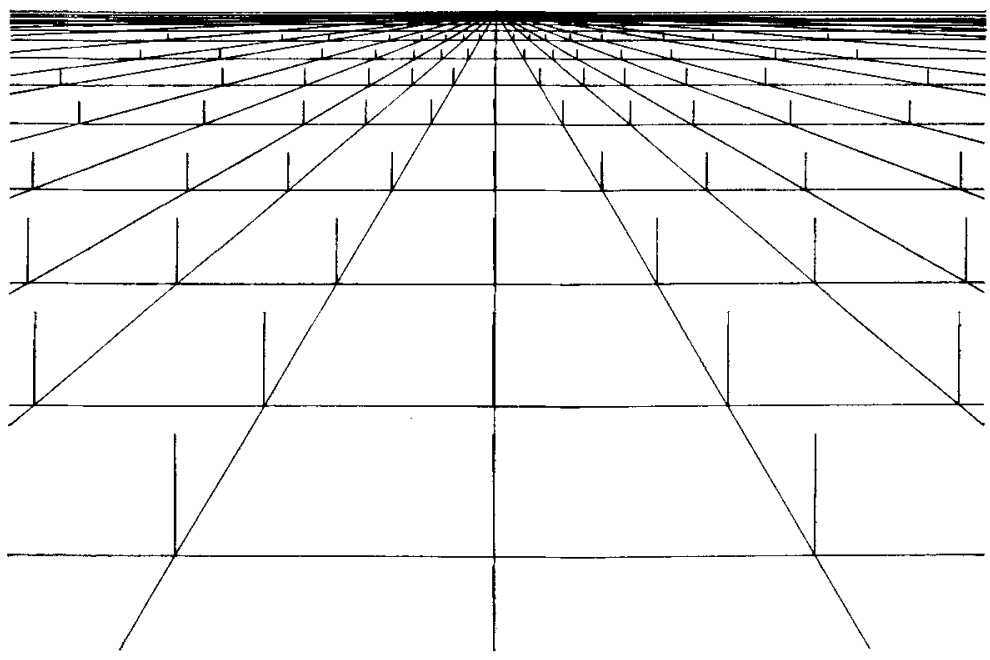

Fig. 1. Perspective gradient upon which disks were superimposed in the "perspective" conditions.

The Instruction groups received the following instructions: "You are to make judgments of the size and distance of objects. We are not interested in the real size and distance of these objects, but in their apparent size and distance. The objects are actually the same size and the same distance from you, but most people see them as different and we are interested in finding out how you perceive them." The No Instruction groups received no preliminary instructions.

All Ss answered the following questions once it was ascertained that they could see both disks. They were instructed to keep both disks in view at all times when making their judgments. Half of the Ss made size estimations first and distance estimations second; the other half made these estimations in the reverse order. The questions designed to elicit phenomenal size estimations were as follows: (1) Do the two targets appear the same size or does one target appear larger than the other? (2) Which target is the smaller, the one lower or higher in your field of vision? (3) Estimate the diameter of the smaller target using any units you wish. (4) If the smaller one appears to be (answer to question (3)) in diameter, how big is the larger one?

The questions designed to elicit phenomenal distance estimations were as follows: (1) Do the two targets appear the same distance away or does one target appear further away than the other? (2) Which target appears closer to you, the one lower or higher in your field of vision? (3) Estimate the distance from you to the closer target using any units you wish. (4) If the closer target appears to be (answer to question (3)) away from you, how far away from you is the further one?

If the response to question (1), for either the size or the distance estimations, was a judgment of equality, then the remaining questions (2), (3), and (4) were omitted.

\section{RESULTS AND DISCUSSION}

Two scores were computed from each $S^{\prime}$ 's estimations. These scores were calculated by dividing the smaller estimate of size, or distance, into the larger estimate of size, or distance. The resulting ratio was considered positive if the circle higher in the S's field of vision was judged as larger than, or further away than the circle lower in the S's fleld of vision; conversely, if the circle lower in the S's field of vision was judged as either larger than, or further away than the circle higher in S's field of vision, then the ratio was assigned a negative value. In order that the scale have zero as the point of equality, rather than the ratio score of 1 , +1 was subtracted from all ratios. Tables 1 and 2 show the means of the distance and size scores, respectively, for all groups.

Separate analyses of variance were performed on the size and distance scores. The size scores for the Perspective groups were reliably larger $(F=11.27$, $\mathrm{df}=1 / 72, \mathrm{p}<.01$ ) than those of the No Perspective groups (whose means closely approximated 0). Similarly, the distance ratios for the Perspective groups were larger than those of the No Perspective groups

Table 1. Mean Distance Scores for All Groups $N=10 /$ cell

\begin{tabular}{lcccccc} 
& \multicolumn{2}{c}{ Instructions } & \multicolumn{2}{c}{ No Instructions } & \\
& Perspective & No-Perspective & Total Mean & Perspective & No-Perspective & Total Mean \\
\hline Size First & 3.36 & -0.13 & 1.62 & 3.27 & 0.02 & 1.65 \\
Distance First & 2.09 & -0.05 & 1.02 & 2.94 & -0.07 & 1.44 \\
Total Mean & 2.73 & -0.09 & 1.32 & 3.11 & -0.03 & 1.54 \\
\hline
\end{tabular}


Table 2. Mean Size Scores for All Groups $N=10 /$ cell

\begin{tabular}{lcccccc} 
& \multicolumn{2}{c}{ Instructions } & \multicolumn{2}{c}{ No Instructions } \\
& Perspective & No-Perspective & Total Mean & Perspective & No-Perspective & Total Mean \\
\hline Size First & 1.40 & 0.15 & 0.78 & 0.53 & 0.03 & 0.28 \\
Distance First & 1.07 & 0.00 & 0.54 & 0.21 & 0.03 & 0.12 \\
Total Mean & 1.24 & 0.08 & 0.66 & 0.37 & 0.03 & 0.20 \\
\hline
\end{tabular}

$(F=13.28, \mathrm{df}=1 / 72, \mathrm{p}<.005)$. Most important, a significant effect was obtained for the Instruction vs the No Instruction groups $(F=4.50, d f=1 / 72, p<.05)$, for the size estimates only.

Orthogonal comparisons revealed that the size estimations for the Instruction-Perspective groups were reliably larger than those for the No InstructionPerspective groups $(p<.05)$. No other orthogonal comparisons within the Perspective or No Perspective groups were significant.

These results confirm the hypothesis that demand characteristics inherent in this situation influenced some Ss toward making objective rather than phenomenal judgments. Also consistent with the stated hypothesis is the fact that none of the observers in the Instruction-Perspective groups gave size judgments of equality and only two gave judgments of equality for the distance judgments. In contrast, four observers in the No Instruction-Perspective groups gave judgments of equality for the size estimations and three for the distance estimations. It should be noted that the distance judgments were not significantly influenced by the instructions, whereas the size judgments were. Perhaps the distance effects in the perspective line drawing were too compelling to be ignored, whereas the size effects were not.
Correlations between size and distance estimations were calculated for the total group and for all subgroups, but no significant correlations were found.

\section{References}

Carlson, V. R. Size-constancy judgments and perceptual compromise. J. exp. Psychol., 1962, 63, 68-73.

Carlson, V. R., \& Tassone, E. P. Independent size judgments at different distances. J. exp. Psychol., 1967, 73, 491-497.

Epstein, W. Attitudes of judgment and the size-distance invariance hypothesis. J. exp. Psychol., 1963, 66, 78-83.

McCready, D. W., Jr. Size-distance perception and accommodationconvergence micropsia - A critique. Vis. Res., 1965, 5, 189-206.

Smith, $\mathbf{0}$. W. Judgments of size and distance in photographs. Amer. J. Psychol., 1958, 71, 529-538.

Smith, O. W., Smith, P. C., \& Hubbard, D. Perceived distance as a function of the method of representing perspective. Amer. $J$. Psychol., 1958, 71, 662-674.

Wohlwill, J. F. The perspective illusion: Perceived size and distance in fields varying in suggested depth, in children and adults. J. exp. Psychol., 1962, 64, 300-310.

\section{Notes}

1. This research was supported by grant number $9425-19$ from the Defense Research Board of Canada.

2. Present address: Department of Psychology, The City College of the City University of New York, New York, N. Y.

(Accepted for publication August 1, 1967.) 